\title{
Laterality differences and practice effects under central backward masking conditions
}

\author{
THOMAS B. WARD and LEONARD E. ROSS \\ University of Wisconsin, Madison, Wisconsin 53706
}

\begin{abstract}
Two experiments were conducted to examine laterality differences and practice effects under various central backward masking conditions. Critical stimulus onset asynchrony (SOA) was determined for subjects on 3 consecutive days using single letters as target stimuli (TS) and a pattern masking stimulus (MS). There was a right visual field (RVF) advantage on Day 1 but no difference between the visual fields on following days. The decline in the RVF advantage appeared to be dependent upon prior experience with laterally located letters, to be independent of initial experience with a particular set of letters, and to be more pronounced for females than for males. In addition, large improvements in performance were found, particularly between the first and second testing sessions. These practice effects were discussed in terms of the possible development of strategies for enhancing TS features or attenuating MS features.
\end{abstract}

Despite an extensive literature demonstrating a right visual field (RVF) left hemisphere advantage in the processing of verbal information (e.g., see Geffen, Bradshaw, \& Wallace, 1971), important questions remain regarding the nature of such laterality differences. The present experiments investigated possible laterality differences in processing speed during a very early stage of information processing, information "read-out" from short-term sensory store, and the extent to which such differences are affected by practice.

Central backward masking procedures have been used to investigate the time needed to process information from short-term sensory store. With this technique, the minimum stimulus onset asynchrony (SOA) between the target stimulus (TS) and a masking stimulus (MS) at which the MS no longer prevents TS identification is determined, with shorter minimum SOAs indicating faster TS processing.

Recently, backward masking paradigms have been used to investigate visual field differences in the processing of briefly presented verbal stimuli. However, in most cases it is unclear to what extent central as opposed to peripheral masking (e.g., Turvey, 1973) was involved, and in no case was the effect of practice on laterality differences examined. Turvey (1973, Experiment 3) found that critical ISIs (interval from TS offset to MS onset) for letters presented to the hemiretinae composing the RVF were shorter than for those same letters presented to the hemiretinae of the LVF. The masking in Turvey's

This research was supported by a National Science Foundation graduate fellowship to the first author and by USPHS Grant HD08240. Reprint requests should be addressed to Leonard E. Ross, Department of Psychology. University of Wisconsin, Madison, Wisconsin 53706. study, however, was of peripheral origin since a random noise mask was used and no masking occurred when the TS and MS were presented to the same hemisphere via different eyes. Oscar-Berman, Goodglass, and Cherlow (1973), using a checkerboard pattern as a MS, found that total processing time for nonalcoholic adults was shorter for words presented to the RVF than for those same stimuli presented to the LVF. The locus of masking in the Oscar-Berman et al. study is unclear, but peripheral masking cannot be ruled out, since their MS field was much brighter than that of the TS and the MS was structually dissimilar to the TS. The only experiments on hemispheric differences in which central masking was clearly achieved with pattern masking were reported in an unpublished doctoral dissertation by Michaels (1973). Michaels examined LVF and RVF dichoptic masking functions using letter trigrams as both TS and MS and found a consistent RVF advantage for the recognition of the trigrams at long (above $40 \mathrm{msec}$ ) but not short SOAs. Laterality differences in metacontrast masking have been reported by McKeever and Suberi (1974), who found that recognition was better for letters in the RVF than in the LVF at most SOA values.

The present experiment was designed to explore laterality differences under pattern (structure) masking conditions found by Turvey (1973) to produce central backward masking. Since none of the previous laterality masking studies examined laterality differences with practice, and since recent experimental work has pointed to the importance of practice effects in visual field differences (Hellige, 1974; Kallman \& Corballis, 1975), an objective of the present series of masking studies was to examine the effects of practice on such differences, as well as on overall performance levels. 


\section{EXPERIMENT 1}

\section{Method .}

Stimuli. Target stimuli consisted of five symmetric letters, A, H, M. T. and Y. The letters were black on white Stenso-Gothic capitals prepared on $35-\mathrm{mm}$ slides. When rear projected onto the "subject's viewing screen, each letter subtended a visual angle of $.8 \mathrm{deg}$ vertical and $.65 \mathrm{deg}$ horizontal. The distance from the near edge of each letter to the fixation point subtended a visual angle of $2 \mathrm{deg}$. The fixation point was indicated by a dot which was approximately one-fourth the height of the letters. The pattern MS. constructed to resemble as closely as possible that used by Turvey (1973). was a random arrangement of variously oriented black bars, equal in width and length to the horizontal and vertical bars of the letters. and subtended a visual angle of $3.9 \mathrm{deg}$ vertical $\times 3.2 \mathrm{deg}$ horizontal. The MS projected to the LVF was identical to that projected to the RVF.

Apparatus. Five Sawyer Rotodisc slide projectors (Model XM), each moditied to contain a Uniblitz shutter (Model 26-A), were used to rear project the stimuli onto the $8 \times 9 \mathrm{~cm}$ screen of a Polymetric eye-movement recorder (Model V-116). The Uniblitz shutters have open and close times of approximately 1-1.5 msec. There was a separate projector for the dot, each MS. the TS, and the lighted background. The luminance of the screen. as measured br a Model J16 Tektronix digital photometer. was 35 nits for the TS and MS: the luminance of the lighted background and fixation dot tields measured 36 and 33 nits, respectively. To avoid any systematic bias due to unequal shutter speed or luminosity differences across the visual field, half of the subjects received the RVF-MS from one projector and the LVF-MS from the other; for the other half of the subjects the visual field/projector relationship was reversed.

The onset and offset of all stimuli were controlled by solid state timing equipment. Eye position was monitored with a Panasonic television camera (Model WV-241P), which was equipped with a silicon-diode tube for sensitivity to low light levels. and a Panasonic television monitor (Model TR-513V). Infrared light-emitting diodes (LEDs) were located at each of the four corners of the screen in the eve-movement apparatus. Since the LEDs delimited the boundaries of the screen. this system permitted relatively easy and fairly precise monitoring of eye position. Subjects viewed all stimuli binocularly. but only the position of the right eye was monitored.

Subjects. Subjects were eight male and eight female undergraduates enrolled in introductory psychology courses at the University of Wisconsin. All had normal or corrected to normal vision. and none had an extreme acuity dominance in either eye (see Procedure section). Subjects received "experimental points" for their participation.

Procedure. Each subject was tested once a day for 3 consecutive davs (sessions). The first session consisted of visual acuity testing, training. and test phases. In the training phase, instructions and practice trials were given. The test phase involved the actual test trials, in which the critical SOA was determined. The second and third sessions consisted of the training and test phases only.

Visual acuity was determined separately for each eye with a Titmus Professional Vision Tester (Model OV-7A). No subject was continued in the experiment who demonstrated a superiority of more than three points in one eye, that is, who judged more than three more stimuli correctly when viewing with one eye than when riewing with the other eye. One subject was replaced on the basis of the ocular dominance criterion. After vision testing, instructions were read to the subjects. The subjects were asked to report, after each trial. which letter was presented, were instructed to fixate the center of the screen from the onset of the dot until after responding, and were discouraged from trying to anticipate the side on which a letter would appear.

Ten practice trials were given with TS, MS. and SOA durations of 150 . 50 , and $350 \mathrm{msec}$. respectively. The experimenter began each test trial with a ready signal. The subject then fixated the dot which appeared in the center of the screen. One and one-half seconds after the dot appeared the subject was briefly shown one of the letters, followed at various intervals by the MS either to the left or right of the dot. The oftset of the dot was synchronous with the onset of the TS. The TS and MS durations, 20 and $50 \mathrm{msec}$. respectively. were chosen to produce central backward masking (Turver. 1973. Experiment 12). The screen was lighted throughout all ISIs and ITIs. Eye position was monitored from the onset of the dot to the onset of the TS so that trials on which fixation was not maintained could be aborted. However. subjects consistently maintained their fixation as instructed.

A procedure quite similar to Turvey's (1973), but with modifications to account for large practice effects within a single experimental session, was used to determine critical SOA. The SOA was initially set at $20 \mathrm{msec}$. and was increased in $5-\mathrm{msec}$ steps until the subject correctly identified four out of five letters at a given SOA. At that point. the SOA was increased in $1-\mathrm{msec}$ steps until the subject correctly identified five letters in succession. If a subject went from a low level of performance to identifying five letters in succession in a 5-msec step. the SOA was decreased until a mistake occurred and increased in $1-\mathrm{msec}$ steps from that point. The TS was changed on every trial whether the subject's response was correct or incorrect. The shortest SOA at which the subject correctly identified five letters in succession was taken to be the critical SOA.

Critical SOAs were determined independently and concurrently for letters on the left and right sides of tixation. The side on which a letter appeared was determined by a Gellerman series. The sequence of letters was randomized. with the restrictions that no letter immediately precede or follow itself and that each letter appear twice. once on each side. in each block of 10 trials. At the end of all testing. the subject filled out a handedness questionnaire (Oldtield. 1971).

\section{Results}

The mean critical SOAs for LVF and RVF presentations for all subjects are plotted in Figure 1 as a function of testing session. A 2 by 2 by 2 by 3 analysis of variance was performed on the critical SOA values, with sex and projector used to present the MS to a visual field as between-subject variables

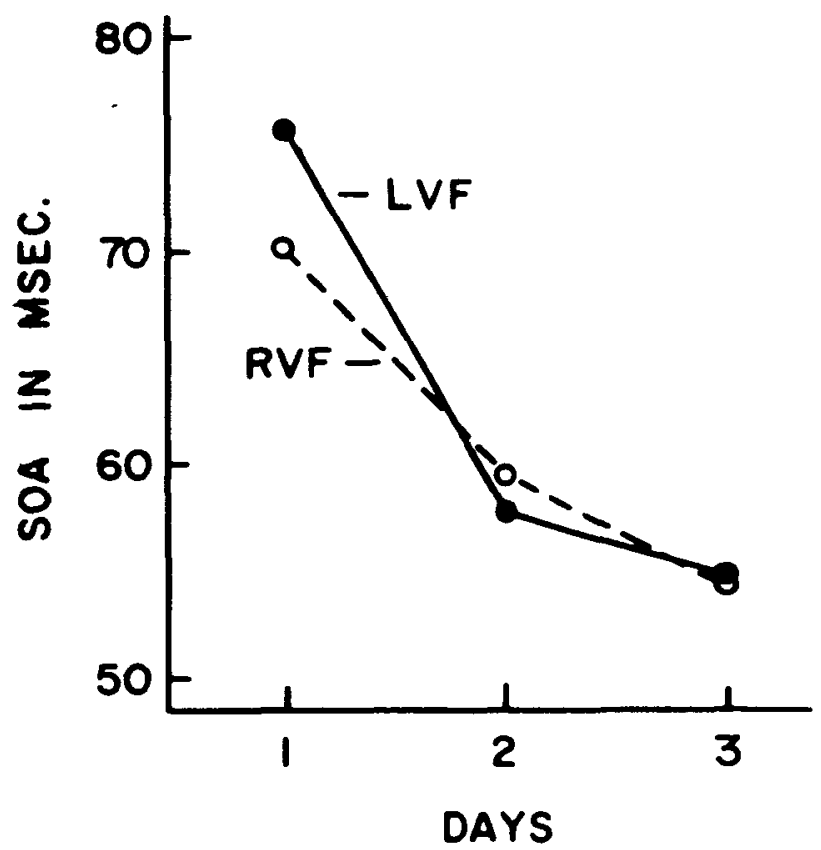

Figure 1. Mean critical SOAs for the LVF and RVF for all subjects as a function of session of testing (days). 
and visual field and sessions as within-subject variables. There were no main effects of sex, projector, or visual field, nor did projector interact with any of the other variables. There was a main effect of sessions $[F(2,24)=44.05, p<.001$, $\mathrm{MSe}=66.79]$, with inspection of Figure 1 revealing that subjects' performance improved dramatically over sessions, regardless of visual field of presentation. Further analysis of the data revealed that Day 1 performance was poorer than either Day $2[\mathrm{t}(15)=6.26, \mathrm{p}<.001, \mathrm{SE}=2.26]$ or Day $3[\mathrm{t}(15)=10.89, \mathrm{p}<.001, \mathrm{SE}=1.68]$. Days 2 and 3 did not significantly differ $[t(15)=2.05$, $\mathrm{p}>.05, \mathrm{SE}=2.01 \mathrm{]}$. There was a significant Visual Field by Sessions interaction $[F(2,24)=15.20$, $\mathrm{p}<.001, \mathrm{MSe}=6.85]$, the nature of which is evident from an inspection of Figure 1. On Day 1, there was a significant advantage, in terms of shorter critical SOAs, for letters presented to the RVF $[\mathrm{t}(15)=5.42, \mathrm{p}<.001, \mathrm{SE}=.99]$, while there was no difference between the visual fields on Day $2[\mathrm{t}(15)=1.15, \mathrm{p}>.20, \mathrm{SE}=1.21]$ or Day 3 $[\mathrm{t}(15)=.52, \mathrm{p}>.20, \mathrm{SE}=1.07]$.

In addition, there was a significant Sex by Visual Field by Sessions interaction $[F(2,24)=3.93$, $\mathrm{p}<.05, \mathrm{MSe}=6.85]$. Although inspection of the data indicated that females showed a more pronounced decline in the RVF advantage than did the males, both groups demonstrated a RVF advantage on Day $1[\mathrm{t}(7)=3.87, \mathrm{p}<.01, \mathrm{SE}=1.39$ for females and $t(7)=5.21, p<.001, S E=1.03$ for males], and neither group demonstrated any significant $(.05$ level) visual field differences on Day 2 or Day 3. However, females showed significantly more improvement in the LVF than in the RVF from Day 1 to Day $2[\mathrm{t}(7)=4.56, \mathrm{p}<.01, \mathrm{SE}=1.92]$, with the reverse being true from Day 2 to Day $3[t(7)=$ $4.54, \mathrm{p}<.01, \mathrm{SE}=1.23 \mathrm{l}$, and males failed to show any differences between the visual fields in terms of amount of improvement. Thus, further tests carried out to analyze the Sex by Visual Field by Session interaction yielded inconsistent results.

Correlation coefficients were computed comparing LVF-RVF SOA differences on Day 1 to a laterality quotient derived from the handedness questionnaire, and LVF-RVF SOA differences on Day 1 to the left-right eye acuity differences as determined by vision testing on Day 1. No important relationships among the variables were found.

\section{EXPERIMENT 2}

Experiment 2 was designed to examine further the nature of the decline in the RVF advantage as demonstrated in Experiment 1. Specifically, Experiment 2 had three purposes. The first was to determine if the decline in the RVF advantage was a replicable finding. The second was to determine if the decline was specific to experience with laterally located letters (i.e., direct stimulation of each hemisphere separately) or if the same effect could be achieved by giving subjects masking experience with centrally located letters. The third objective was to determine if the effect was specific to the letter set with which the subject had initial experience.

\section{Method}

Apparatus and stimuli. The apparatus and stimuli were the same as those described in Experiment 1, except that an additional set of letters, $O, U, V, W$, and $X$ was used. The $O$ and $U$ were composed of straight line parts of other letters to avoid any possible bias in recognition of the curved portions of those letters.

Subjects. Twelve male and 12 female subjects, paid for their services, served in Experiment 2. All had normal or corrected to normal vision, and none had an extreme acuity dominance as defined in Experiment 1.

Procedure. As in Experiment 1, subjects were tested on 3 consecutive days, with visual acuity testing, training, and test trials on Day 1 and training and test trials on Days 2 and 3 . Day 1 stimuli consisted of letters located either centrally or laterally, with the letters being either the same set as on Days 2 and 3 ( $A, H, M, T, Y)$ or a different set $(O, U, V, W, X)$. Crossing these factors resulted in four Day 1 test conditions: central-same, central-different, lateral-same, and lateral-different.

Six subjects were randomly assigned to each of the four Day 1 test conditions, with the restriction that there be equal numbers of males and females in each group. For all subjects, Days 2 and 3 were the same as Days 2 and 3 of Experiment 1, that is, SOAs were determined for the letter set $A, H, M, T$, and $Y$, located either to the left or right of fixation. It should be noted that the lateral-same condition is essentially a replication of Experiment 1.

A slightly different method was used in Experiment 2 to determine critical SOA. It was determined that children. with whom the present procedures were to be used in future research, could not tolerate repeated failure to see the TS during the initial test trials of the short-SOA-first (increasing) procedure of Experiment 1. Accordingly, a long-SOA-first (decreasing) procedure was employed in Experiment 2 so that future developmental research could be carried out with comparable procedures. In the decreasing procedure, the SOA was initially set at $120 \mathrm{msec}$, a value at which no subject had difficulty responding with the correct letter. The SOA was decreased from that point until the critical SOA was reached. Ward (1975, Experiment 2) compared these procedures and found them to yield comparable data.

Since determination of SOAs for the LVF and RVF separately could result in twice as many trials as a single determination for centrally located letters, critical SOAs were determined twice on Day 1 for subjects in the central conditions in order to equate the number of Day 1 trials across conditions. Projector-mask position was not a significant source of variance in Experiment 1, therefore this variable was not manipulated in Experiment 2.

\section{Results}

Figure 2 presents the mean critical SOAs for subjects in all four experimental conditions, averaged across sex of subject as a function of testing session.

Replication of Experiment 1. An analysis of variance using only the data from subjects in the Day 1 lateral conditions replicated the Experiment 1 sessions effect and Visual Field by Sessions interaction $\quad[F(2,16)=62.33, \quad \mathrm{p}<.001, \quad \mathrm{MSe}=$ 45.93 and $F(2,16)=5.26, \quad p<.05$, $\mathrm{MSe}=6.62$, respectively]. As can be seen by 

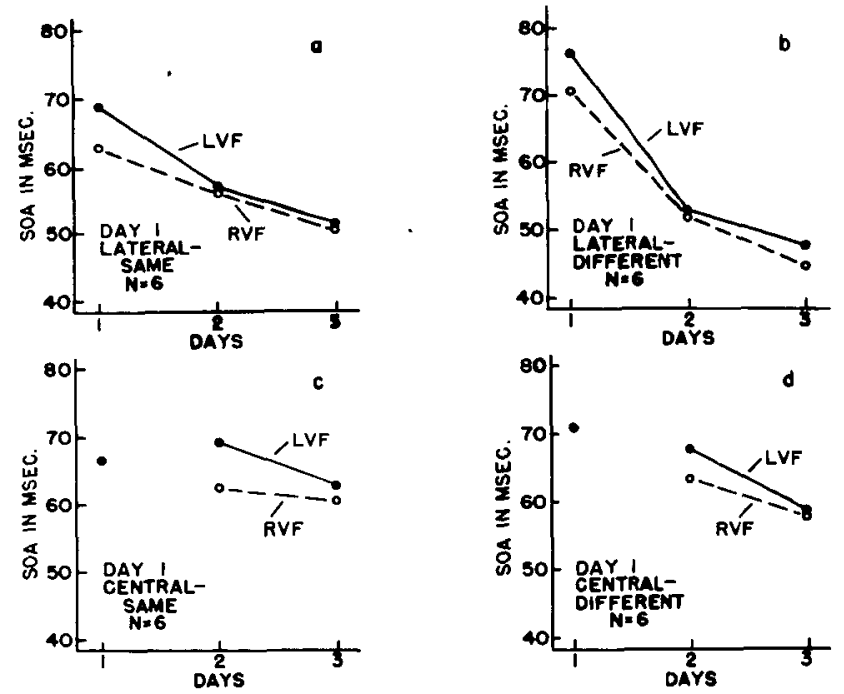

Figure 2. Mean critical SOAs for subjects in all conditions as a function of session of testing. The single unconnected dots in Figures $2 \mathrm{c}$ and $2 \mathrm{~d}$ represent Day 1 performance averaged over first and second SOA determinations for subjects in the central-same and central-different conditions, respectively.

comparing Figures $2 a$ and $2 b$ with Figure 1, the Visual Field by Sessions interaction was of the same form, with a decreasing RVF advantage, in both experiments. Also as in Experiment 1, there was no main effect of visual field of presentation $[\mathrm{F}(1,16)=2.82, \mathrm{p}>.10, \mathrm{MSe}=52.33]$. Although the Sex by Visual Field by Sessions interaction fell just short of significance at the .05 level $[F(2,16)=3.48$, $\mathrm{p}<.10, \quad \mathrm{MSe}=6.62]$, inspection of the data revealed a pattern consistent with the results of Experiment 1. One difference between the two experiments was the finding of a significant overall improvement in performance from Day 2 to Day 3 $[\mathrm{t}(11)=3.17, \mathrm{p}<.01, \mathrm{SE}=1.72]$ in Experiment 2.

Central vs lateral conditions. Comparison of Figures $2 a$ and $2 b$ with $2 c$ and $2 d$ reveals the effect of Day 1 experience with laterally located vs centrally located letters on the RVF advantage. It can be seen that the RVF advantage was negligible on Days 2 and 3 following Day 1 lateral presentations but present on Day 2 following Day 1 central presentations. In the latter case, however, the RVF advantage then declined after Day 2 sessions during which the subject experienced laterally located letters. Statistical support for these observations is found in an analysis of variance conducted on the Day 2 and Day 3 critical SOAs for subjects in all groups. Sex, Day 1 letter set, and Day 1 letter location (central vs lateral) were between-subject variables, while sessions, Days 2 and 3 , and visual field of presentation were within-subject variables. The analysis revealed a significant Letter Location by Sessions by Visual Field interaction $[\mathrm{F}(1,16)=4.58, \mathrm{p}<.05, \mathrm{MSe}=9.60]$. Subsequent $t$ tests revealed that in the lateral conditions there was no difference between the visual fields on Days 2 $[\mathrm{t}(11)=.55, \mathrm{p}>.20, \mathrm{SE}=1.82]$ or $3[\mathrm{t}(11)=$ $1.10, \mathrm{p}>.20, \mathrm{SE}=1.82$ ], while in the central conditions there was a significant RVF advantage on Day $2[\mathrm{t}(11)=2.36, \mathrm{p}<.05, \mathrm{SE}=2.54]$ but not on Day $3[\mathrm{t}(11)=.72, \mathrm{p}>.20, \mathrm{SE}=2.20]$. Thus, it appears that previous experience with laterally located letters is necessary for reduction in the RVF advantage to occur in later sessions.

Comparison of Figures $2 a$ and $2 b$ with $2 c$ and $2 d$ also reveals that there was greater overall improvement from Day 1 to Day 2 for those receiving lateral presentations on Day 1 as compared to those receiving centraily located letters on Day 1 . This observation is supported by a second analysis on the performance of all groups across all three sessions without consideration of visual field differences (since subjects in the Day 1 central conditions did not have separate scores for the RVF and LVF on Day 1, laterality effects could not be examined in a comparison of all groups across all 3 days). The analysis revealed a significant Day 1 Letter Location (central vs lateral) by Sessions interaction $\quad[F(2,32)=6.79, \quad \mathrm{p}<.005$, $\mathrm{MSe}=44.65]$, with the lateral and central conditions having nearly identical SOAs on Day 1 but the lateral condition groups showing more improvement than the central condition groups as revealed by shorter SOAs on Day $2[\mathrm{t}(11)=2.92, \mathrm{p}<.01, \mathrm{SE}=4.00]$ and Day $3[\mathrm{t}(11)=2.28 . \mathrm{p}<.05 . \mathrm{SE}=5.14]$. Consistent with this observation, the analysis of the data from Days 2 and 3 revealed only a significant main effect of Day 1 letter location $[F(1,16)=5.92$, $\mathrm{p}<.05, \mathrm{MSe}=554.18]$, with those in the Day 1 lateral conditions performing better on Days 2 and 3 than those who received the central condition on Day 1. Thus, there was more improvement when lateral presentations followed lateral presentations than when they followed central presentations.

Same vs different letter set conditions. Comparison of Figures $2 \mathrm{a}$ and $2 \mathrm{c}$ with $2 \mathrm{~b}$ and $2 \mathrm{~d}$ reveals that Day 1 same or different letter set had little effect in determining laterality differences or changes in those differences on subsequent days. In the previously described analysis comparing laterality performance across Days 2 and 3, there was no main effect of Day 1 letter set and no significant Visual Field by Day 1 Letter Set or Visual Field by Day 1 Letter Set by Sessions interactions (all Fs $<1$ ), with MSes of $554.18,52.89$, and 9.60 , respectively, for the comparisons. Thus, it is clear that the letter set experienced on Day 1 had little or no effect on observed laterality differences or changes in those differences.

Comparison of Figures $2 a$ and $2 c$ with $2 b$ and $2 d$ also reveals that there was as much or more improvement across sessions for those subjects receiving different letters on Day 1 as there was for those receiving the same letters on all days. In fact, Day 1 letter set interacted significantly with sessions $[\mathrm{F}(2,32)=3.75, \quad \mathrm{p}<.05, \quad \mathrm{MSe}=44.65] \quad$ when 
performance across all 3 days was considered. Performance was slightly, although not significantly, better for those in the same letter set condition than for those in the different letter set condition on Day 1 , but the reverse was true on Days 2 and 3, a pattern that indicates more improvement for those subjects receiving the different letter set on Day 1. Apparently, then, Day 1 experience with the letter set $O, U, V, W$, and $X$ was at least as beneficial to overall Day 2 performance on the letter set $\mathrm{A}, \mathrm{H}, \mathrm{M}, \mathrm{T}$, and $\mathrm{Y}$ as was Day 1 experience with that letter set itself.

In addition to the results reported above, both the analyses of the data from all sessions and of the data from Days 2 and 3 revealed significant main effects of sessions $[F(2,32)=30.62, \mathrm{p}<.001, \mathrm{MSe}=44.65$ and $F(1,16)=21.77, \quad \mathrm{p}<.001 . \quad \mathrm{MSe}=36.18$, respectively]. Subsequent $t$ tests revealed improvement, significant at the .001 level, from Day 1 to Day 2 and from Day 2 to Day 3, with SEs of 2.39 and 1.33, respectively. Neither analysis revealed a main effect of sex, and there was no significant main effect of visual field of presentation in the analysis concerned with laterality effects $[F(1,16)=3.18$, $\mathrm{p}>.05, \mathrm{MSe}=52.89]$.

Correlations between laterality as determined by questionnaire and acuity dominance and laterality as reflected by a RVF advantage on Day 1 failed to reveal any important relationships.

\section{DISCUSSION}

\section{Visual Field Differences}

Both experiments clearly show a small but consistent RVF advantage on Day 1, a finding that is consistent with previous laterality research that employed backward masking techniques (McKeever \& Suberi, 1974; Michaels, 1973; Oscar-Berman et al., 1973; Turvey, 1973). Given the assumption that the critical SOA in central backward masking reflects the time needed for information to be processed from sensory information store, the present results suggest that the left hemisphere advantage for the processing of verbal information is present, at least initially, to some extent as early in the information processing sequence as the stage of read-out from sensory store.

The most interesting aspect of the laterality data is not the RVF advantage found on Day 1, however, but the combination of that finding with the lack of visual field differences on following days. Since this interaction appeared in both Experiments 1 and 2 and with both the increasing and decreasing procedures, it is probably not a spurious one. Further, the fact that in Experiment 2 there was significant overall improvement in performance from Day 2 to Day 3, with no RVF advantages on Day 2, indicates that floor effects were not involved.

From the present data, two conclusions can be drawn regarding the nature of the reduction in the
RVF advantage: It is independent of the specific letter set initially experienced, and experience with laterally located letters is necessary for the reduction to occur. The second conclusion follows from the fact that the reduction occurred on the day subsequent to the first experience with laterally located letters, that is, Day 2 for the lateral groups and Day 3 for the central groups.

Changes in hemispheric differences over sessions in situations not involving masking have been reported by Hellige (1974) and Kallman and Corballis (1975). An explanation offered by Kallman and Corballis to account for their data was that the hemisphere which normally performs a task can become "fatigued," allowing the contralateral hemisphere to increase its processing load, thus reducing the initial laterality advantage. Since a decline in the RVF advantage was observed in the present experiments after a period of $24 \mathrm{~h}$, such fatigue is an unlikely explanation for the present results.

Regardless of the ultimate interpretation of the present data, it is clear that explanations of visual field differences that are based on fixed structural and functional characteristics of the hemispheres and do not allow for changes in observed differences over sessions are incomplete. Models which consider processes whereby observed laterality effects can change (see e.g., Kinsbourne, 1973) seem more promising in view of the present data and that reported by Hellige (1974) and Kallman and Corballis (1975).

An interesting aspect of the laterality data is that females showed a more pronounced decline in the RVF advantage from Day 1 to Day 2 than did the males. This pattern was present in both experiments, although the Sex by Visual Field by Sessions interaction failed to reach statistical significance at the .05 level in Experiment 2. While the result is intriguing, particularly in view of other recent reports of sex interactions in laterality studies (see, e.g., Metzger \& Antes, 1976), any interpretation of such interactions based on the present data would be highly speculative.

\section{Practice Effects}

One of the most prominent features of the present data, which is consistent with previous masking research (e.g., Schiller, 1965; Turvey, 1973, Experiment 10 ), is the improvement in performance that occurred with practice. In the present study, practice effects were found when letters were either centrally or laterally located, when Day 1 experience was with letters that were the same or different than those presented in later sessions, and when either an increasing or decreasing method was used to determine the critical SOA. There are a number of possible interpretations of these practice effects. One is that subjects show a nonspecific adaptation to the experimental situation. That is, the relative novelty of 
the masking situation may depress subjects' performance for any number of reasons (e.g., disruption of attention). Thus, the improvement might represent a general adjustment to such novelty. Another interpretation is that subjects develop specific strategies for processing the presented information more efficiently. Data favoring the second interpretation over the first comes from work of Turvey (1973, Experiment 10 and Footnote 14) and Schiller (1965), both of whom reported the existence of practice effects in central but not in peripheral masking. If improvement over trials was a matter of general adaptation to the experimental situation, then practice effects might be expected in both types of masking.

If the practice effects in central backward masking do represent developing strategies, at least three possibilities can be suggested: Subjects might "set" themselves to pick out or enhance certain distinctive features of the TS, learn to avoid or postpone processing the irrelevant information in the MS, or use both processes in combination. The fact that subjects receiving different letters on Day 1 than on following days showed as much, or more, improvement as those receiving the same letters on all days suggests either that the strategies were not specific to the particular letter set presented or that, if they were letter set specific, they developed rapidly during the experimental session. The finding that subjects receiving letters in lateral locations on all days showed more improvement than those receiving letters in central locations on Day 1 indicates that some type of location-specific transfer may have occurred, however.

The notion of subjects being able to enhance certain features and attenuate others is not new. For example, Egeth (1967), in reviewing the work of Neisser and others on visual search, pointed out that subjects may be thought to set themselves to selectively process the critical features of the target. Thus, practiced subjects do not "see" distractor items when searching, but pass over them in a blur until the target item seems to jump out at them. Egeth suggested that the masking of a stimulus in visual masking situations might represent the subject's inability to filter the relevant and irrelevant stimulus attributes in such a manner. If this is the case, improvement in performance might represent the development of strategies for more efficient filtering, such as enhancing certain critical TS features or attenuating MS features.
While the particular mechanisms or strategies involved are not identified, the laterality and practice effects and their interaction found in the present data emphasize the possible importance of set and strategy factors in hemispheric specialization and backward masking. Consequently, caution should be exercised when interpreting laterality and backward masking differences in groups differing in practice, age, or intellectual level, where such set and strategy factors might be differentially utilized.

\section{REFERENCES}

Egeth, H. Selective attention. Psychological Bulletin, 1967, 67, 41.57 .

Geffen, G., Bradshaw, J. L., \& Wallace, G. Interhemispheric effects on reaction time to verbal and nonverbal visual stimuli. Joumal of Experimental Psychology, 1971, 87, 415-422.

Hellige, J. B. Hemispheric processing differences revealed by differential conditioning and reaction time performance. Unpublished doctoral dissertation, University of Wisconsin, 1974.

Kallman, H. J., \& Corballis, M. C. Ear asymmetry in reaction time to musical sounds. Perception \& Psychophysics, 1975, 17, 368-370.

KINSBOURNe, M. The control of attention by interaction between the cerebral hemispheres. In $S$. Kornblum (Ed.), Attention and performance IV. New York: Academic Press, 1973.

MCKeever, W. F., \& Suberi, M. Parallel but temporally displaced visual half field metacontrast functions. Quarterly Journal of Experimental Psychology, 1974, 26, 258-265.

Metzger, R. L., \& ANres, J. R. Sex and coding strategy effects on reaction time to hemispheric probes. Memory \& Cognition, 1976, 4, 167-171.

Michaels, C. F. An examination of hemispheric asymmetry in the visual processing of linguistic items. Unpublished doctoral dissertation, University of Connecticut, 1973.

OldfiELd, R. C. The assessment of handedness: The Edinburgh inventory. Neuropsychologia, 1971, 9, 97-111.

Oscar-Berman, M., Goodglass, H., \& Cherlow, D. C. Perceptual laterality and iconic recognition of visual materials by Korsakoff patients and normal adults. Journal of Comparative and Physiological Psychology, 1973, 82, 316-321.

Schiller, P. H. Monoptic and dichoptic visual masking by patterns and flashes. Journal of Experimental Psychology. 1965, 69, 193-199.

Turvey, M. T. On peripheral and central processes in vision: Inferences from an information-processing analysis of masking with patterned stimuli. Psychological Review, $1973,80,1-52$.

WARD, T. B. Laterality differences and practice effects in central backward masking. Unpublished Masters thesis, University of Wisconsin, 1975.

(Received for publication August 4, 1976; revision received October $18,1976$. 